\title{
Eye movements during visual search for artistically embedded targets
}

\author{
CALVIN F. NODINE, DENNIS P. CARMODY and EDWARD HERMAN \\ Radiology Research Laboratory, Temple University School of Medicine, \\ Philadelphia, Pennsylvania 19140
}

\begin{abstract}
Eye movements were measured as subjects searched for a word target embedded in line drawings. Time taken to find the target was directly related to the degree of embeddedness or target conspicuity. Detections were predicted by multiple-fixation clusters exceeding 400 msec within $3 \mathrm{deg}$ of the target. Search errors resulted from faulty pattern recognition in which the target was fixated, but for dwell times shorter than the 400-msec detection threshold found for hits. Dwell time is taken to reflect recognition and decision processes used to discriminate real from potential targets.
\end{abstract}

Several search tasks have shown that the conspicuity of the target, defined as the degree to which the target is embedded in the surround, directly influences target detection (Kundel \& Nodine, 1978; Kundel, Revesz, \& Graber, 1974). Embedded targets, because they have more features in common with the surround and therefore blend into it, are harder to find than nonembedded targets that stand out from the surround.

The targets (the word NINA) in artist Al Hirschfeld's drawings, are noted for their lack of conspicuity because most of the features of the word are constructed of the same lines used to make up the picture. Finding NINAs is both entertaining and instructive, because it shows how the artist effectively uses the principle of embedding to hide targets in an artistically rendered scene. In fact, and paradoxically, the "background" in which NINAs are embedded is usally considered the "foreground" from both the artist's and the viewer's point of view, and the NINA targets are synonymous with Hirschfeld's signature. Other artists have also experimented with the principle of embedding to hide figures in their art works. One of the best known examples is Salvador Dali's painting, "The Slave Market with Disappearing Bust of Voltaire."

From these examples, it is clear that the artist has discovered that the principle of embedding depends on the interrelationship between target and background. The greater the overlap between these two feature sets, the greater the degree of embedding.

In a similar way, military targets are embedded in scenic backgrounds using another invention of the artist: camouflage. In this case, the principle of embedding may include not only overlap of target and back-

This research was supported by DAAG Grant 29-76-G-0313. Portions of this paper were presented at the 18th Annual Meeting of the Psychonomic Society, Washington, D.C., November 1977. Thanks to Drs. H.L. Kundel and B.F. Nodine and D.F. Fisher for reading and criticizing the manuscript. ground features, but also occlusion of the target by the background as, for example, the camouflaged tank parked under a tree. This principle of camouflage not only works in favor of the artist and against the viewer in scenic displays (Behrens, 1978), but also in favor of the prey and against the predator in nature (Hinton, 1973), and in favor of the disease and against the radiologist in medicine (Kundel \& Nodine, 1978). In all cases, the conspicuity of the target is influenced by the degree to which the target is embedded in the surround.

If embedded targets are harder to find because they have more features in common with the background than do nonembedded targets, then reducing the amount of overlap between target and background should improve search performance and provide concommitant changes in eye movement parameters like fixation cluster patterns or dwell time (Nodine, Carmody, \& Kundel, 1978). To test these hypotheses, we took the liberty of disembedding NINA targets by transplanting them to other less dense backgrounds. Operationally, this manipulation produced three levels of embedding: the original rendition by the artist, in which overlap was considered high; our reembedding of the NINA targets in less densely lined surrounds, where overlap with the target was reduced; and, finally, transplantation of the NINA target to a clear background to produce a nonembedded target.

\section{METHOD}

\section{Stimulus Materials}

Four drawings were selected from The World of Hirschfeld (Hirschfeld, 1970) to test our predictions: "Inherit the Wind," "Miracle Worker," "Elmer Gantry," and "Night of the Iguana."

We photographically transplanted the NINA target from each of these drawings to another densely lined area of the scene or to a clear area. Thus, there were three versions of embedded NINAs for each of the four pictures. Figure 1 illustrates how we manipulated degree of embedding for the picture "Elmer Gantry." To illustrate, there are three degrees of embedded NINA in this picture. 


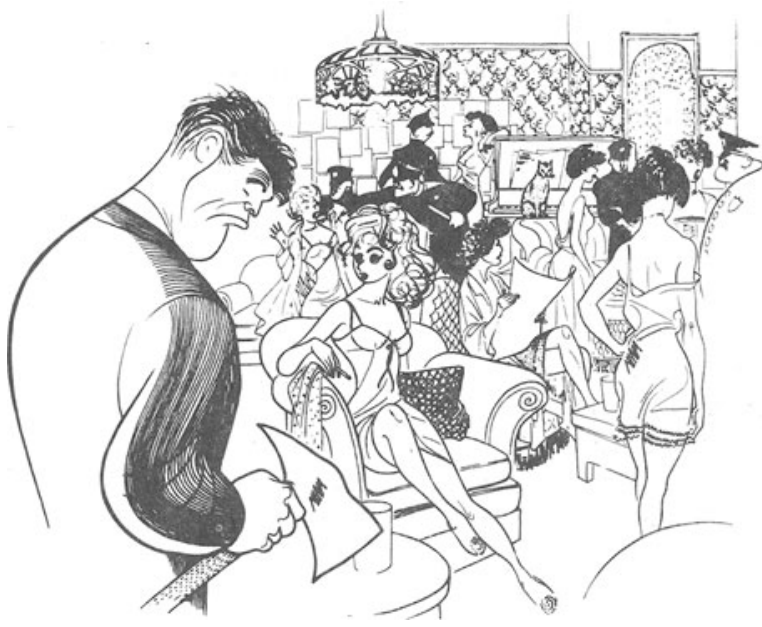

Figure 1. Scene from "Elmer Gantry" showing three different levels of embedded NINA target. The original Hirschfeld NINA is embedded in gown of woman in left background. The reembedded NINA is in the folds of the slip of the woman in the right foreground. The nonembedded NINA is on Burt Lancaster's axe. Subjects saw only one NINA target per picture. (Reproduced from Hirschfeld, 1970, p. 102).

\section{Procedure}

Ten adult subjects, five experienced finding NINA and five inexperienced, saw three versions of four pictures for a total of 12 search trials. Orders were randomized between subjects. Eye movements were recorded as the subjects searched for NINA targets using a Biometrics eye movement monitor interfaced to a PDP-11/40 computer. Pictures were rear-projected onto a screen $70 \mathrm{~cm}$ in front of subjects who viewed the pictures while their heads were held by a combination head-and-chin rest. Prior to and following the viewing of each picture, subjects fixated a 25-point calibration pattern arranged in a 5 by 5 array that was used to measure and subsequently correct errors due to irregularities in the surface of the cornea or misalignment of the sensors. After the subject fixated the last point on the calibration array, a second projector presented a fixation point for $2 \mathrm{sec}$, which served as the starting point for search. The picture followed for $10 \mathrm{sec}$, or until the target was detected, in which case the subject pushed a button that terminated the display and caused the calibration pattern to reappear. The subject refixated five points on the calibration pattern, the four corners and the center, in order to measure head movement that might have occurred during the task. Records in which head movement exceeded $3 \mathrm{deg}$ were discarded. Of 120 eye movement records, 96 met the pre/postcalibration criterion. The reliability of these 96 calibration records showed an average pre/post difference of $1.15 \mathrm{~cm}$ (slightly less than $1 \mathrm{deg}$ ) with a standard deviation of $.48 \mathrm{~cm}$. Further, the discarded records were distributed evenly over experimental conditions.

Because fixations could not confidently $(95 \%)$ be resolved more accurately than $\pm 2 \mathrm{deg}$ and the size of the NINA targets was approximately $1 \mathrm{deg}$, for purposes of analysis we scored a fixation as falling on the target if it fell within a 3-deg radius of the target center. The projected pictures were slightly larger than the 5 by 5 calibration array.

\section{RESULTS AND DISCUSSION}

Search time, accuracy, and eye movements were recorded, and these data were subjected to ANOVA with type of embeddedness as a within-subjects effect and subject experience as a between-subjects effect. Re- cording eye movements during the search task was necessary to determine dwell time, that is, the total fixation time (usually the result of mulitple fixations) spent in the 3-deg target zone. Previous search tasks for NINA targets (Nodine et al., 1978) and for nodules in radiographs (Kundel, Nodine, \& Carmody, 1978) showed that dwell times in the target zone exceeding $400 \mathrm{msec}$ signaled correct detections with a high probability.

In the present experiment, the average dwell time for a correct detection was over twice that for a miss $(613 \mathrm{msec}$ vs. $282 \mathrm{msec}) \quad[\mathrm{F}(1,6)=36.25, \mathrm{p}<.01]$, and longer dwell was associated with nearly twice as many fixations for correct detections (3.25) as for misses (1.75) $[F(1,6)=12.60, p<.01]$, confirming previous findings regarding the usefulness of the dwell time as a measure of cognitive processing. Applying a 95\% confidence band around the mean dwell for hits $(613 \mathrm{msec})$ yields a predicted lower limit for a hit of $400 \mathrm{msec}$ (-2 SDs).

As shown in Table 1 , between $60 \%$ and $76 \%$ of the correct detections, depending on degree of embedding, showed dwell times $\geqslant 400 \mathrm{msec}$. A relatively small proportion of misses ranging from $8 \%$ to $36 \%$ showed dwell times $\geqslant 400 \mathrm{msec}$. Thus, dwell times greater than $400 \mathrm{msec}$ are likely to signal the detection of a potential NINA target. Whether this longer dwell resulted in a correct detection or a miss depended on the subject's ability to recognize features that differentiated the NINA target from the surround, for example, the " $A$ " in the NINA (Nodine et al., 1978). These distinctive target features should be easier to recognize for nonembedded NINAs than for either original or reembedded NINAs. As a result, nonembedded NINAs should be easier to find and require less dwell time than either type of embedded NINA. This was the case. Average dwell times were significantly longer for original NINAs (564 msec) than either reembedded (396 msec) or nonembedded $(383 \mathrm{msec})$ NINAs $[\mathrm{F}(2,12)=3.62$, $\mathrm{p}<.05$ ]

Experienced subjects found almost twice as many NINAs $(.65)$ as inexperienced subjects $(.37)[\mathrm{F}(1,24)=$ $9.42, \mathrm{p}<.01]$. This relationship was consistent across levels of embedding. As predicted, both groups found more nonembedded NINAs (.63) than either reembedded $(.46)$ or original $(.43)$ NINAs $[F(2,48)=4.82, p<.05]$.

Somewhat surprising was the finding that $90 \%$ of the misses resulted from failures to fixate the NINA target for sufficient dwell time, which for our task was

Table 1

Proportion of Fixation Clusters $\geqslant \mathbf{4 0 0}$ msec for Hits and Misses and as a Function of Degree of Embedding

\begin{tabular}{lccc}
\hline & \multicolumn{3}{c}{ Degree of Target Embedding } \\
\cline { 2 - 4 } & Original & Reembedded & Nonembedded \\
\hline Hits & .76 & .68 & .60 \\
Misses & .36 & .23 & .08 \\
\hline
\end{tabular}


below a detection threshold of $400 \mathrm{msec}$, and not from failure to fixate the target zone. The fact that average dwell time for misses was shorter than the threshold dwell but longer than the average dwell of all fixations (mean $=237 \mathrm{msec}$ ) and the fact that nonembedded targets were found faster than original or reembedded targets suggest that some pictorial feature attracted the subject's attention and hence influenced search. Perhaps it was a novel or ambiguous scenic feature, perhaps it was even a target feature.

Just what constitutes a novel or ambiguous feature has been the subject of much speculation. With line drawings it could be the detection of changes in the texture, gradient, or line of depicted scenic features that match a distinctive target feature (Gibson, 1966). It could be the selection and testing of scenic features that fit the subject's perceptual schemata for disambiguating the target from the background (Neisser, 1976). Whichever the case, the subject failed to recognize the cue as a potential target feature and thus missed the target.

\section{Fixation Patterns}

Figure 2 shows the fixation patterns of two subjects looking at "Elmer Gantry." The subject on the left detected the original NINA target with a cluster of five fixations (numbers 10-14) for a total dwell of $800 \mathrm{msec}$. The subject on the right fixated the target zone three times but failed to detect the target. On two of the three occasions (Fixations 3 and 20), the subject fixated the target for less than $400 \mathrm{msec}$, indicating a pattern recognition error. Between these two fixations, the subject fixated the target zone for $450 \mathrm{msec}$ (Fixations 1315). Clearly, on this occasion, the subject saw something within the target zone that was interesting enough to capture his attention, but his analysis of the pattern led to an erroneous decision. Thus, failures to find NINA targets during search can be attributed primarily to faulty recognition or decision processes and not to failures of the eye to sample the target site.

There is another difference in Figure 2 between the eye movement pattern on the left, which resulted in a correct detection, and the one on the right, which resulted in a miss. In addition to fixating the zone containing the original NINA target, the subject on the left fixated both other target sites where he had previously found reembedded (Fixation 4) and nonembedded (Fixations 7-8) NINA targets. A further check of fixations on sites previously found to contain NINA targets revealed that this occurred in $43 \%$ of the cases, and that it was equally common among experienced and naive subjects. This tendency for subjects to search sites where targets had previously been found indicates the important role memory exercises on eye movement patterns during visual search.

\section{Attracted Eye vs. Intelligent Eye Models of Search}

The fixation cluster prior to target detection has significance in visual search according to either an "attracted-eye" model or an "intelligent-eye" model. The attracted-eye model says that the eye is drawn to certain distinctive features (e.g., As rather than Is). The detection of these distinctive visual features depends primarily on peripheral guidance of eye movements. Thus, this model predicts that eye movement events upstream from the point at which the target was detected play a role in search. We expected differences in saccade length between hits, where, presumably, peripheral vision cued detection prior to fixating the target,

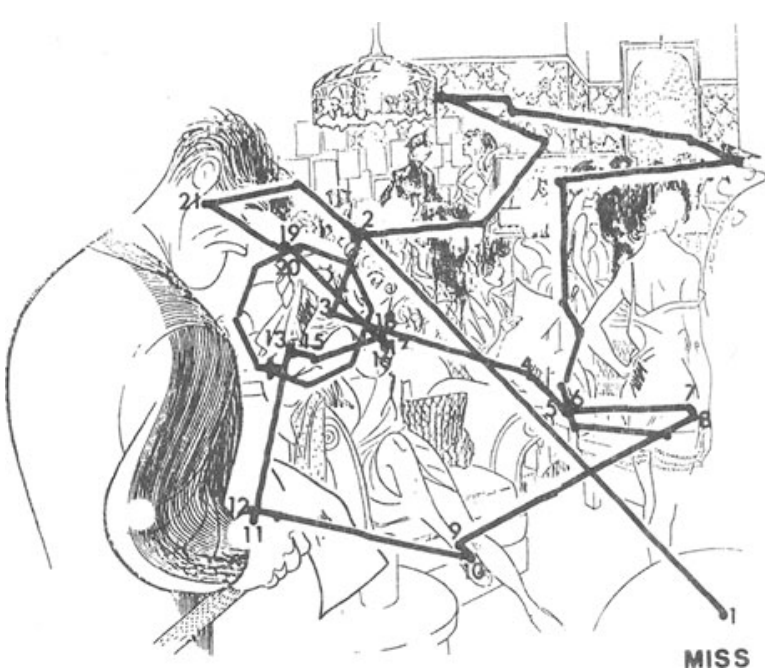

Figure 2. (Left) Shows the fixation pattern of a subject that correctly detected the original NINA target on the gown of the woman in the left background. A 3-deg circle indicates the target zone. Five fixations fell within this zone for a total dwell time of $800 \mathrm{msec}$. The calibration pattern is superimposed over the picture to show the size of the display. Calibration points are 5 deg apart. This subject also fixated both previously detected NINA targets, which are also shown. (Right) Shows fixation pattern of subject who failed to detect the same NINA target, even though he fixated within the target zone on three separate occasions. This subject used the entire $10 \mathrm{sec}$ of search time. The subject on the left found the target within $2 \mathrm{sec}$. 
and misses, where peripheral cuing failed to detect target features. No differences were found between hits and misses (when the target was fixated) in three analyses of eye movement events upstream from the point at which the target was fixated: (1) dwell time of the fixation cluster prior to fixating the target, (2) number of fixations in this prior fixation cluster, and (3) length of the saccade (jump) from the last fixation of the prior cluster to the first fixation on the target. Also, the attracted-eye model predicts that differences in search performance are due primarily to scanning errors, that is, failure of the eye to sample the target zone. This accounted for only $10 \%$ of the search errors.

The intelligent-eye model stresses the role of cognitive expectancies, built up by prior experience with the task, to direct the eye to potential target sites. This model predicts that subjects will systematically eliminate potential sites based on probabilities generated from prior experiences finding NINA targets. Highprobability target sites include such pictorial features as wisps of hair and folds of clothing, We have found that these features are examined during search, but there is no difference between experienced and naive subjects in terms of search time to a correct detection.

Our results favor the intelligent-eye model, which predicts that search performance depends primarily on the subject's ability to recognize both potential target sites and target features. Such recognition ability, according to the model, is tied to a "target hypothesis" (after Gregory's, 1970, object hypothesis), which spells out the ways the NINA will appear when embedded in a pictorial surround. Thus, the emphasis in our search model is on an understanding of pictorial features likely to hide a target and the set of potential transformations that a target can assume when combined with the pictorial features. We believe that this knowledge is essential for finding embedded targets in Hirschfeld's search task.

\section{CONCLUSIONS}

Some important conclusions result from this study. First, we have shown that making NINA targets more or less conspicuous by manipulating degree of embedding in the surround affects search performance. Second, dwell time is a useful eye movement paramter for differentiating hits from misses during search. The data suggest a detection threshold of $400 \mathrm{msec}$. Probability of target detection is high when dwell on the target zone exceeds this threshold, while shorter dwell times on the target zone are likely to result in misses; these are called pattern recognition errors. A small percentage of misses are caused by failures to sample the target zone with the eye; these are sampling errors. Third, failure to sample the target zone with the eye can result in hits. About a quarter of the hits in the present study fell outside a target zone radius of $3 \mathrm{deg}$. These hits are interpreted as resulting from peripheral vision, suggesting that the useful field of view may extend as far as \pm 5 deg during search for NINA targets. Fourth, memory for previously detected targets influences eye movement patterns.

\section{REFERENCES}

Behrens, R. R. On the visual art of camouflage. Leonardo, 1978, 11, 203-204.

Gibson, J. J. The senses considered as perceptual systems. Boston: Houghton-Mifflin, 1966.

Gregory, R. L. The intelligent eye. New York: McGraw-Hill, 1970.

Hinton, H. E. Natural deception. In R. L. Gregory \& E. H. Gombrich (Eds.), Illusion in nature and art. London: Duckworth, 1973.

HirsChFELD, A. The world of Hirschfeld. New York: Abrams, 1970.

Kundel, H. L., \& Nodine, C. F. Studies of eye movements and visual search in radiology. In J. W. Senders, D. F. Fisher, \& R. A. Monty (Eds.), Eye movements and the higher psychological processes. Hillsdale, N.J: Erlbaum, 1978.

Kundel, H. L., Nodine, C. F., \& CARmody, D. P. Visual scanning, pattern recognition and decision-making in pulmonary nodule detection. Investigative Radiology, 1978, 13, 175-181.

Kundel, H. L., Revesz, G., \& Graber, M. A. The influence of structured noise on the detection of radiologic abnormalities. Investigative Radiology, 1974, 9, 479-486.

NeISSER, U. Cognition and reality. San Francisco: Freeman, 1976.

Nodine, C. F., CARMody, D. P., \& Kundel, H. L. Searching for NINA. In J. W. Senders, D. F. Fisher, \& R. A. Monty (Eds.), Eye movements and the higher psychological processes. Hillsdale, N.J: Erlbaum, 1978.

(Received for publication February 23, 1979.) 